Research Journal of Medical Sciences 6 (1): 22-25, 2012

ISSN: $1815-9346$

(C) Medwell Journals, 2012

\title{
Micro Leakage Assessment of Pit and Fissure Sealant with Previous Fluoride Application: An in vitro Study
}

\author{
${ }^{1}$ Mahkameh Mirkarimi, ${ }^{2}$ Mahdieh Beheshti and ${ }^{1}$ Fatemeh Mahmoudi \\ ${ }^{1}$ Department of Pediatric Dentistry, Zahedan University of Medical Sciences, Zahedan, Iran \\ ${ }^{2}$ Department of Pedodontist, Tehran, Iran
}

\begin{abstract}
Adhesion of restorative materials to the tooth structure is significance in dentistry. The aim of this study was to evaluate the micro leakage of pit and fissure sealants following application of $1.23 \%$ Acidulate Phosphate Fluoride (APF). Thirty sound non carious human premolar teeth were randomly assigned in two groups $(\mathrm{n}=20$ ). Artificial fissure grooves were prepared on the buccal surface of each tooth. In test group $1.23 \% \mathrm{APF}$ gel was applied for $4 \mathrm{~min}$ to thoroughly dried teeth and then teeth etched $15 \mathrm{sec}$ using phosphoric acid gel. Bonding was applied to the fissures and polymerized with a light curing unit and the fissure sealant material (3M, USA) were placed on the fissures. In control group the procedure was the same as test group but with the omission of the fluoride application. All the teeth were thermo cycled 1000 times at 5-55oc with dwell time of $30 \mathrm{sec}$. Samples were immersed in $2 \%$ basic fuchsine solution for $24 \mathrm{~h}$ and the teeth were sectioned buccolingully for the middle of the fissure. Dye penetration was evaluated under stereomicroscope. In test group $20 \%$ of the specimens and $46.7 \%$ of those in the control group shows no dye penetration. The dye had penetrated to the base of the fissure in $26.7 \%$ of test group and $20 \%$ of controls. Comparing groups by Mann Whitney U-test revealed that the difference between groups was not significant $(\mathrm{p}<0.05)$. The results reveal that the use of topical fluoride treatment immediately prior to sealant placement does not increase micro leakage.
\end{abstract}

Key words: Micro leakage, fluoride, fissure sealant, penetration, treatment, bonding, Iran

\section{INTRODUCTION}

Fissure sealants have been widely used from 1960s for control of occlusal caries (Mejare et al., 2003). If the fissures are sealed completely and micro leakage is prevented they act as a physical barrier to various external carious agents thus preventing the onset of caries (Mejare et al., 2003; Shaw, 2000).

Adhesion of restorative materials to tooth structure is one of the greatest problems in dentistry. If the lack of adhesion exists between resin restoration and enamel the micro leakage occurs and it leads to discoloration, secondary caries and marginal breakdown. The method is technique sensitive in which many factors intervene and modify the effects.

On the other hand application of topical fluoride agents in children is well accepted as a clinical preventive procedure.

The use of fluoride prior to sealant placement is controversial for many decades. It has been suggested that application of fluoride prior to tooth etching lowers the tensile bond strength (Low et al., 1975; Sheykholeslam et al., 1972) but other studies have suggested that fluoride does not adversely affect bond strength of enamel with composites or sealants (Warren et al., 2001; Koh et al., 1995; Takahashi et al., 1980).

Furthermore the application of the sealant material over a fluoride treated surface should provide prolonged contact of the fluoride with the enamel and enhance the uptake (Takahashi et al., 1980).

Another fact to consider is that fluoride applications reduce the surface energy of enamel and it interferes with the wetting of the tooth surface by a liquid adhesive (MacDonald, 2011). To solve these contradictory results further investigations is required.

The aim of this study is to evaluate the micro leakage of pit and fissure sealants following application of $1.23 \%$ acidulated phosphate fluoride. It was believed that the findings would have potentially important implications for clinical use of sealants.

\section{MATERIALS AND METHODS}

Thirty sound non carious human maxillary and mandibular premolar teeth which were extracted for orthodontic or periodontal reasons were collected. Teeth were brushed and washed then, stored in $0.5 \%$

Corresponding Author: Mahkameh Mirkarimi, Department of Pediatric Dentistry, Zahedan University of Medical Sciences, Zahedan, Iran 


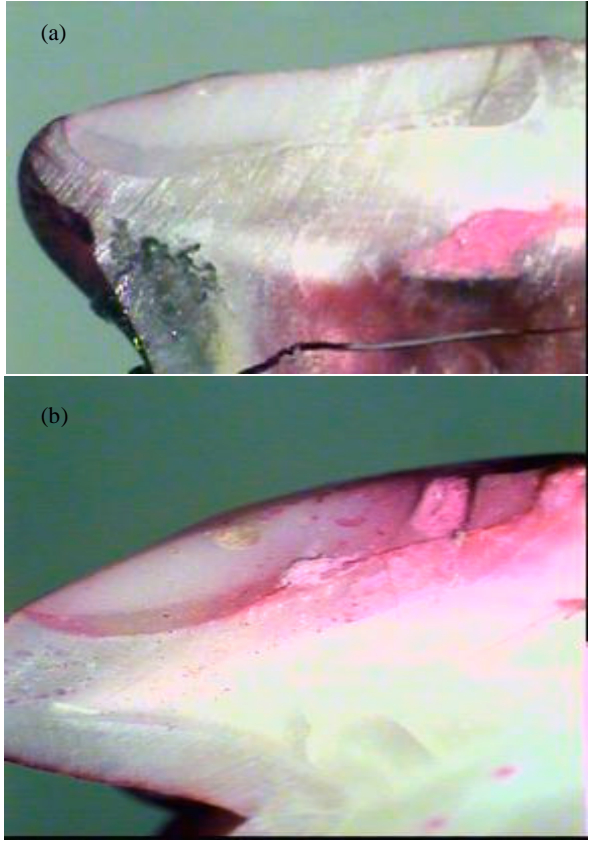

Fig. 1: Dye penetration evaluation under stereomicroscope (a: Grade 0, b: Grade 3)

chloramines solution for $24 \mathrm{~h}$ and kept in saline until the beginning the experiment. Two layers of nail polish were applied to coat the teeth approximately $2 \mathrm{~mm}$ from the margins of the sealant. Teeth were mounted in a self curing acrylic base by using metallic molds to allow ease in handling. Artificial fissure grooves (Width $1 \mathrm{~mm}$ ) confined to a depth of $1 \mathrm{~mm}$ in enamel were then prepared on the buccal surface of each tooth using a $1 / 4$ round bar (D and $Z$, Germany) with a high speed hand piece.

The teeth were divided in to two groups using simple randomization method. In test group $1.23 \%$ APF gel (Sultan, USA) was applied for $4 \mathrm{~min}$ to thoroughly dried teeth and then teeth etched $15 \mathrm{sec}$ using $37 \%$ phosphoric acid gel (3M, USA), washed $30 \mathrm{sec}$ and dried for $15 \mathrm{sec}$. A layer of bonding agent ( $\mathrm{N}$ Bond, Germany) was applied to the fissures and dried gently for $5 \mathrm{sec}$ with compressed air and polymerized with a light curing unit.

Sealant (3M, ESPE, USA) was applied and light cured according to the instructions of the manufacture and the margins of sealants were then checked for any failure of sealant application. In control group the procedure was the same as test group but with the omission of the fluoride application. All the teeth were thermo cycled 1000 times at $5-550 \mathrm{oc}$ with dwell time of $30 \mathrm{sec}$. Samples were immersed in $2 \%$ basic fuchsine solution for $24 \mathrm{~h}$ and then washed. The teeth were sectioned buccolingully for the middle of the fissure using a disk mounted on a slow speed hand piece. One blinded examiner was evaluated the dye penetration (Fig. 1a and b) according to the bellows criteria under stereomicroscope (Zervou et al., 2000):

$0=$ No dye penetration

$1=$ Dye penetration restricted to the outer half of the sealant

$2=$ Dye penetration extending to the inner half of the sealant

3 = Dye penetration extending to the underlying fissure

The results were statistically evaluated and the comparisons of the groups were performed using Mann Whitney U-test and a value of $\mathrm{p}<0.05$ was considered significant.

\section{RESULTS AND DISCUSSION}

The percentage of teeth that showed micro leakage in each group is shown in Table 1. In test group $3(20 \%)$ of the specimens and $7(46.7 \%)$ of those in the control group shows no dye penetration. The dye had penetrated to the base of the fissure in $4(26.7 \%)$ of test group and $3(20 \%)$ of controls.

Comparing groups by Mann Whitney U test revealed that the difference between groups was not significant $(\mathrm{p}<0.05)$.

One of the most important factors for success of pit and fissure sealant treatments is the integrity of tooth sealant material interface.

Although, micro leakage can be expected on all restorative materials however, various materials and preparation techniques have been suggested to decrease or even prevent micro leakage. It's clear that the repeated use of fluorides is one of most important roles for prevention of dental caries.

Acidulated Phosphate Fluoride (APF) solution includes acids for the etching of the enamel and the consequent enhancement of the fluoride uptake (Lee et al., 2010).

The results of this study supports that use of fluoride prior to sealant placement does not increased micro leakage. These finding confirms previous studies (Warren et al., 2001; Takahashi et al., 1980; Garcia-Goday et al., 1991). On the other hand Low et al. (1975) reported that APF applied prior to tooth etching lowered the tensile bond strength and they strongly recommended that clinical procedures which involve etching of the enamel should not be preceded by the topical application of APF solutions. 
Table 1: Micro leakage values in two groups

\begin{tabular}{|c|c|c|c|c|c|}
\hline \multirow[b]{2}{*}{ Flouride } & \multicolumn{5}{|l|}{ Leak } \\
\hline & 0.00 & 1.00 & 2.00 & 3.00 & Total \\
\hline \multicolumn{6}{|l|}{$\overline{\text { Yes }}$} \\
\hline Count & 3.0 & 2.0 & 6.0 & 4.0 & 15.0 \\
\hline Within flouride (\%) & 20.0 & 13.3 & 40.0 & 26.7 & 100.0 \\
\hline \multicolumn{6}{|l|}{ No } \\
\hline Count & 7.0 & 3.0 & 2.0 & 3.0 & 15.0 \\
\hline Within flouride (\%) & 46.7 & 20.0 & 13.3 & 20.0 & 100.0 \\
\hline \multicolumn{6}{|l|}{ Total } \\
\hline Count & 10.0 & 5.0 & 8.0 & 7.0 & 30.0 \\
\hline Within flouride (\%) & 33.3 & 16.7 & 26.7 & 23.3 & 100.0 \\
\hline
\end{tabular}

In this manner Sheykholeslam et al. (1972) indicated that topical treatment of ground etched enamel with fluoride, prior to application of self curing resin adhesives resulted in significant reduction in bond strength. It should be point out that in the mentioned studies they have used the ultraviolet light activated resins and this kind of resins are no longer used. It seems that the developments have occurred in the sealant materials during the time and they may shows better clinical properties.

To prevent the influence of the anatomical structure of the fissures and complex morphology of occlusal pits which affects the clinical success of the sealant (Eronat et al., 2003) in this study artificial fissure grooves were prepared on the buccal surface of teeth. Some studies indicated using of bonding agents after etching process is unnecessary and does not affect their clinical effectiveness (Boksman et al., 1993), on the other hand there are other studies which support that bonding agents provides adequate bond strength and retention for resin sealants (MacDonald, 2011) so, in this study the researchers have used bonding agent which was thoroughly air dried across the surface to avoid a thick layer of adhesive residue.

In this study micro leakage was investigated using a dye leakage model with basic fuchsine. This technique has been used in many studies to evaluating micro leakage. It has been believed that if a material responds positively to in vitro dye tests it is likely to respond even better clinically because the dye is more easily diffused than bacteria and their by products (Toman et al., 2007). There are advantages to placing a sealant after a topical fluoride treatment in clinical practice. If the sealant becomes totally or partially lost tooth structure underneath sealants would have benefit of fluoride.

Tandon and Mathew (1997) stated that acid etched enamel treated with $\mathrm{APF}$ remineralized at a more rapid rate also patients having received a fluoride therapy at same visit would not need to be rescheduled for sealant placement.

\section{CONCLUSION}

The results reveal that the use of topical fluoride treatment immediately prior to sealant placement does not increase micro leakage and there was no apparent different permeability of fissure sealants with or without previous fluoride application.

\section{ACKNOWLEDGEMENT}

The researchers acknowledge Mohammad Javad Kharazifard for assistance with the statistical analyses.

\section{REFERENCES}

Boksman, L., R.J. McConnel, B. Carson and E.F. McCutcheon-Jones, 1993. A 2 years clinical evaluation of two pit and fissure sealants placed with and without the use of a bonding agent. Quintessence Int., 24: 131-133.

Eronat, N., Y. Bardakci and M. Sipahi, 2003. Effects of different preparation techniques on the microleakage of compomer and resin fissure sealants. J. Dent. Child, 70: 250-253.

Garcia-Goday, F., R. Perez and G.W. Hubbard, 1991. Effect on prophylaxis pastes on shear bond strength. J. Clin. Orthod., 25: 571-573.

Koh, S.H., Y.Y. Huo, J.M. Powers and J.T. Chan, 1995. Topical fluoride treatment has no clinical effect on retention of pit and fissure sealants. J. Gt. Houst. Dent. Soc., 67: 16-18.

Lee, Y.E., H.J. Baek, Y.H. Choi, S.H. Jeong, Y.D. Park and K.B. Song, 2010. Comparison of remineralization effect of three topical fluoride regimens on enamel initial carious lesions. J. Dent., 38: 166-171.

Low, T., J.A. Von Fraunhofer and G.B. Winter, 1975. The bonding of a polymeric fissure sealant to topical fluride treated teeth. J. Oral Rehab., 2: 303-307.

MacDonald, R.E., 2011. Dentistry for the Child and Adolescent. 9th Edn., Mosby Publishing Co., St. Louis, MO., USA., Pages: 42.

Mejare, I., P. Lingstrom, L.G. Petersson, A.K. Holm and S. Twetman et al., 2003. Caries preventive effect of fissure sealants: A systematic review. Acta Odontol. Scand., 61: 321-330.

Shaw, L., 2000. Modern thoughts on fissure sealants. Dent. Update, 27: 370-374.

Sheykholeslam, Z., M.G. Buonocore and A.J. Gwinnett, 1972. Effects of fluorides on the bonding of resins to phosphoric acid etched bovine enamel. Arch. Oral Biol., 17: 1037-1045.

Takahashi, Y., Y. Arakwa, T. Matsukubo and M. Takeuchi, 1980. The effect of sodium fluoride in acid etching solution on sealant bond and fluoride uptake. J. Dent. Res., 59: 625-630. 
Tandon, S. and T.A. Mathew, 1997. Effect of acid etching on fluoride treated caries like lesion of enamel: A SEM study. J. Dent. Child, 64: 344-348.

Toman, M., S. Toksavul, C. Artunc, M. Turkun, P. Schmage and I. Nergiz, 2007. Influence of luting agent on microleakage of all-ceramic crowns. J. Adhes Dent., 9: 39-47.
Warren, D.P., N.B. Infante, H.C. Rice, S.D. Turner and J.T. Chan, 2001. Effect of topical fluoride on retention of pit and fissure sealants. J. Dent. Hyg., 75: 21-24.

Zervou, C., E.H. Doherty, A. Zavras and G.E. White, 2000. An in vitro study of microleakage of pit and fissure sealants in the presence of occlusal forces. J. Clin. Pediatr. Dent., 24: 273-278. 